\title{
Características de la intervención ocupacional en un caso de trastorno del espectro autista
}

\author{
Characteristics of an occupational intervention in a case \\ of autism spectrum disorder
}

\author{
Angélica Monsalve Robayo ${ }^{1}$ \\ Nathalia Alejandra Cubillos Forero ${ }^{2}$ \\ Maira Lizeth Peña Duarte ${ }^{3}$ \\ Angela María Peñaranda Saavedra ${ }^{4}$
}

Recibido: 9 diciembre 2014 • Enviado para modificación: 27 mayo 2015 • Aceptado: 27 junio 2015

Monsalve, A., Cubillos, N. A., Peña, M.L. \& Peñaranda, A.M. (2015). Características de la intervención ocupacional en un caso de trastorno del espectro autista. Revista Ocupación Humana, 15 (1), pp. 5 - 18.

Resumen: Se reporta la intervención ocupacional realizada durante dos meses y medio en el servicio de Terapia Ocupacional de la IPS Quinta Mutis- Universidad del Rosario en Bogotá, Colombia, con un niño de 3 años y 9 meses de edad, diagnosticado con trastorno del espectro autista. El DSM-5 clasifica este diagnóstico dentro de los trastornos del neurodesarrollo, caracterizado por dificultades en la comunicación, la interacción social, los patrones repetitivos de comportamiento e intereses limitados. La metodología utilizada incluyó la evaluación a través de la entrevista a padres, la aplicación del instrumento propio del servicio y de la escala del desarrollo de Merrill Palmer. La intervención se fundamentó en los principios de los enfoques de integración sensorial, adaptación espacio temporal y el modelo de habilidades adaptativas; e incluyó actividades lúdicas de carácter motor y sensorial en ambientes estructurados y no estructurados. Se encontraron avances significativos en destrezas de regulación emocional, cognitivas, de comunicación y sociales, que impactaron en su desempeño familiar y escolar. Se concluye que la intervención dirigida a la modulación sensorial, la autorregulación, el seguimiento de instrucciones, los procesos atencionales y la interacción con pares, es fundamental para lograr avances en el desempeño ocupacional del niño.

Palabras clave: Trastorno del espectro autista, niños, Terapia Ocupacional.

Abstract: This report presents the occupational intervention of a 3 year- 9 month old boy diagnosed with autism spectrum disorder. The child received Occupational Therapy services for a 2-month period at the IPS Quinta Mutis of the Universidad del Rosario in Bogota, Colombia. The DSM-5 classifies this diagnosis amongst the neurodevelopmental disorders. It is characterized by difficulties in communication, social interaction, repetitive patterns of behavior, and limited interests. The methodology included the assessment of the child's development through: an interview with the parents, a global occupational therapy evaluation

\footnotetext{
1 Terapeuta Ocupacional. Profesora de Terapia Ocupacional. Universidad del Rosario, Bogotá, Colombia. angelica.monsalve@urosario.edu.co

${ }^{2}$ Estudiante de Terapia Ocupacional. Universidad del Rosario. cubillos.Nathalia@urosario.edu.co

${ }^{3}$ Estudiante de Terapia Ocupacional. Universidad el Rosario.pena.maira@urosario.edu.co

${ }^{4}$ Terapeuta Ocupacional. Especialista en Desarrollo Infantil y Procesos de Aprendizaje. Profesora de Terapia Ocupacional. Universidad del Rosario. penaranda.angela@urosario.edu.co
} 
specific to the institution, and the use of Merrill-Palmer scales of development. The intervention was based on the principles of the sensory integration approach, the spatiotemporal adaptation model, and the adaptive skills model. Intervention strategies included motor and sensory playbased activities in structured and unstructured environments. Results show significant progress in emotional, cognitive, communication, and social regulation skills, with a positive impact on his family relations and his school performance. We conclude that interventions focused to improve sensory modulation, self-regulation, following directions, attention processes, and interaction with peers, are essential for progressing the child's performance.

Key words: Autistim spectrum disorder, children, Occupational Therapy.

\section{Antecedentes y propósito}

El presente reporte busca contribuir al conocimiento de profesionales de la salud sobre las características de la intervención de Terapia Ocupacional, a partir del caso de un niño diagnosticado con trastorno del espectro autista (TEA). Dado que los hallazgos en las diferentes bases de datos reportan intervenciones ocupacionales en otras latitudes diferentes a Colombia, este reporte de caso se justifica en la necesidad de documentar el uso de estrategias evaluativas y procedimientos de intervención ocupacional con un niño y su familia, para contribuir al desarrollo científico de la profesión en el país.

Se describen entonces el proceso de evaluación, la utilización de referentes teóricos que orientan la realización de actividades con propósito, la participación de la familia y la importancia de la interdisciplinariedad en Terapia Ocupacional. Las autoras muestran los resultados del proceso y los avances logrados tras 20 sesiones de trabajo.

Matsushima \& Kato (2013) afirman que el trastorno del espectro autista está caracterizado por dificultades en la interacción social y la comunicación, así como la presencia de comportamientos estereotipados e intereses limitados. Por otra parte, mencionan que las personas con TEA presentan diferencias significativas en las respuestas sensoriales; estas impactan su atención, su participación e interacción con el entorno y su praxis, evidenciada en una escasa capacidad de imitación y de representación sensorial del movimiento y secuencia motora.

Para el profesional de Terapia Ocupacional es fundamental conocer las características clínicas del TEA. Así pues, es necesario tomar en cuenta criterios diagnósticos para este trastorno, tales como los dados por la quinta edición del Manual Diagnóstico y Estadístico de los Trastornos Mentales, de la Asociación Americana de Psiquiatría (DSM-5), y la Clasificación Internacional de Enfermedades (CIE-10). Estos presentan una visión clara acerca de las dificultades y los componentes neurológicos afectados en el TEA.

De acuerdo con el DSM-5, el TEA se agrupa dentro de los trastornos del neurodesarrollo, cumpliendo, entre otros, con los siguientes criterios: déficits persistentes en la comunicación 
y la interacción social, con alteración de los comportamientos verbales y no verbales, manifestándose en la persona durante su desarrollo; patrones restrictivos y repetitivos de comportamiento, intereses o actividades, caracterizados por la utilización de objetos o la realización de movimientos de manera repetitiva, inflexibilidad en rutinas e intereses restringidos, la ausencia de juego espontáneo, imitativo o imaginativo (Asociación Americana de Psiquiatría, 2014).

Según la CIE-10, el autismo se clasifica dentro del trastorno generalizado del desarrollo, definido por la presencia de un desarrollo alterado o anormal. Se describe como un trastorno proveniente de un daño o disfunción en los procesos neuro-anatómicos, los cuales se presentan en el lapso de los primeros 30 meses de edad, en donde sucede la maduración de circuitos neuronales. Dicha disfunción se manifiesta en alteraciones que influyen en el adecuado desarrollo del cerebro, lo que perjudica principalmente las funciones cognitivas, el desarrollo del lenguaje, las destrezas de regulación emocional y las habilidades de interacción - comunicación. Lo anterior se observa en la incapacidad de relacionarse con personas de su misma edad y de compartir con estos sus intereses y metas. Por otra parte, se detecta la presencia de conductas motoras repetitivas, estereotipias e intereses inusuales y peculiares (Talero, Rodríguez, De La Rosa, Morales \& Velez-Van-Meerbeke, 2012).

Con relación al componente neurológico afectado en el TEA, autores como Rivero \& Garrido (2014) afirman que la alteración en la sustancia blanca de las vías fronto-temporales, se relaciona con la dificultad que presentan estos individuos en sus habilidades sociales y comunicativas; su capacidad para afrontar situaciones nuevas; su limitación para explorar actividades de interés; dificultades en su memoria verbal, flexibilidad cognitiva; y con los trastornos de atención que suelen estar presentes. Además, se observan algunas alteraciones en el hemisferio derecho relacionadas con la dificultades en la comprensión, la expresión facial, el contacto visual y otras características del lenguaje no verbal. Matsushima \& Kato (2013) reportan que los déficits neurológicos dentro del sistema límbico de personas con autismo, pueden crear limitaciones en la motivación.

Según Mulas et al. (2010), el tratamiento del individuo con TEA comprende la utilización de medicamentos tales como naltrexona, secretina o ACTH, para controlar los síntomas y las manifestaciones del desorden en la conducta de la persona. Por otra parte, existen intervenciones conductuales con el fin de enseñar a los niños y niñas comportamientos y habilidades nuevas y adaptativas, e intervenciones evolutivas para promover en el individuo relaciones positivas y significativas con su entorno. El involucramiento del terapeuta ocupacional en el tratamiento, comprende educar a padres o cuidadores generando una participación de asociación, la cual, afirma Arnstein (1971), les permite compartir responsabilidades, tomar decisiones y empoderarse durante el proceso. Además, se utilizan estrategias como la integración sensorial, la estimulación sensorial y la formación en el autocui- 
dado, entre otras, para el desarrollo, compensación o restablecimiento de habilidades afectadas como lo son las de interacción-comunicación y cognitivas (Howell, Wittman, \& Bundy, 2012).

\section{Descripción del caso}

JPJS, de 3 años y 9 meses de edad, nace el 21 de enero de 2011 en la ciudad de Bogotá, en la semana 38 de gestación, por medio de cesárea de emergencia cuando su madre tenía 40 años de edad; la cesárea se realiza ya que su madre refirió no sentir al bebé. Al nacer, JPJS presentó un peso de 3.550 gr, una talla de $52 \mathrm{~cm}$ y membrana hialina ${ }^{5}$, por lo cual le fue necesario permanecer 27 días con oxígeno suplementario en una unidad de cuidados intensivos y, posteriormente, en una unidad de cuidados intermedios. A los dos meses de nacido, JPJS sufrió bronquiolitis con varias repeticiones, por lo que requirió hospitalizaciones frecuentes durantes los primeros meses de vida. El niño recibió lactancia hasta los 6 meses, asumió y mantuvo posición sedente sin apoyo a los 7 meses, gateó a los 11 meses, marchó con apoyo a los 13 meses y sin apoyo a los 17 meses.

Su familia está constituida por papá, mamá y JPJS, no tiene hermanos y es cuidado principalmente por los dos padres. No existen antecedentes familiares importantes a nivel clínico.

Es remitido desde Neuropediatría, quien diagnostica TEA y trastorno del desarrollo del lenguaje el 3 de septiembre de 2013; JPJS ingresa por primera vez al servicio de Fonoaudiología con 2 años y 6 meses de edad, posteriormente es remitido a Terapia Ocupacional, en donde se realiza la apertura de su historia ocupacional, se firma el consentimiento informado y es evaluado el 8 de abril de 2014 .

\section{Primera impresión clínica}

Dados los antecedentes de JPJS y la revisión de la evidencia alrededor del tema, es preciso documentar este caso para contribuir al estudio sistemático de la intervención de Terapia Ocupacional con niños y niñas con TEA y sus familias en Colombia. La inquietud y la oportunidad de conocer el nivel de desarrollo del niño a partir de la observación clínica y la aplicación de una escala que involucra actividades participativas, resulta de capital interés. El caso llama además la atención, en tanto permitiría conocer el nivel de desarrollo y el potencial existente del niño para iniciar un proceso de intervención ocupacional fundamentado en integración sensorial, adaptación espacio temporal y habilidades adaptativas, las cuales, de acuerdo a lo reportado por los padres del niño, estaban alteradas.

\section{Evaluación}

El proceso de evaluación incluye la entrevista a padres, la observación clínica y la aplicación de la Escala de Desarrollo Merrill Palmer (Roid \&

\footnotetext{
${ }^{5}$ La enfermedad de la membrana hialina se caracteriza por una dificultad respiratoria progresiva de aparición precoz, habitualmente desde el nacimiento o en las primeras seis horas de vida. Se produce por falta de surfactante pulmonar, sustancia que tiene como función reducir las fuerzas de tensión superficial de los alvéolos y así mantener la estabilidad y volumen de los pulmones en la espiración (Ventura-Junca \& Tapia, 2004).
} 
Sampers, 2004). En el momento de dicha evaluación, JPJS tenía 3 años y 3 meses de edad. A partir de la entrevista a sus padres, se conoce que la familia del niño está conformada por su padre y madre, sin otros hermanos/as. Sus padres reportan la necesidad de supervisar y ayudar a JPJS de manera permanente en actividades de la vida cotidiana, en especial en el vestido, la alimentación y el baño. Por otro lado, manifiestan que a su hijo le interesa más jugar de manera solitaria que involucrarse en actividades lúdicas con otros niños de su edad. Refieren que JPJS se comunica con dificultad, pero logra expresar sus necesidades y deseos. Les preocupa que no realiza contacto visual y que abandona rápidamente las tareas que inicia.

Para la observación clínica se utiliza el Protocolo del Laboratorio de Juego y Aprendizaje (Peñaranda, 2007), el cual requiere de maniobras y actividades estructuradas para observar las destrezas ocupacionales del niño a nivel motor grueso, motor fino, sensoriointegrativas, cognitivo-perceptuales y de interacción-comunicación. La aplicación de este instrumento tuvo una duración de 60 minutos distribuidos en dos sesiones de 30 minutos cada una. Se complementa el proceso con la batería de evaluación de Escalas de Desarrollo de Merrill Palmer, la cual se aplica durante dos sesiones, cada una de 35 minutos (Roid \& Sampers, 2004). La finalidad de esta escala de desarrollo es evaluar cinco áreas principales del desarrollo: la cognición, el lenguaje, la motricidad, la conducta adaptativa y el autocuidado, y el área socioemocional. Los hallazgos del pro- ceso evaluativo inicial y posterior a la intervención se presentan en el apartado de resultados.

\section{Segunda impresión clínica}

Teniendo en cuenta los resultados de la evaluación inicial, se hace necesario identificar en la evidencia los diferentes enfoques de intervención que han sido utilizados para trabajar con niños y niñas que presentan dificultades de interacción y limitación en el desarrollo de destrezas sensorio-integrativas y cognitivo-perceptuales. Por tanto, se decide utilizar el enfoque de integración sensorial, el enfoque de adaptación espacio temporal y el modelo de habilidades adaptativas, reconociendo sus postulados teóricos y los aportes que brindan al proceso terapéutico en materia de utilización del ambiente físico como posibilitador de experiencias positivas para el alcance de objetivos, el direccionamiento del plan de acción y la relación niño-terapeutafamilia.

\section{Intervención}

En el proceso de intervención se establece como meta: promover respuestas adaptativas frente a demandas del entorno, que le permitan al niño descubrir habilidades, sentir placer y divertirse al desempeñar su rol familiar, escolar y social. A partir de esto, se determinaron como objetivos del plan de tratamiento:

- Generar conciencia corporal y autorregulación a través de experiencias sensoriales, especialmente con estímulos táctiles, propioceptivos y vestibulares. 
- Lograr el seguimiento de tres a cuatro instrucciones de manera consecutiva, estableciendo contacto visual en la ejecución de circuitos motores.

- Estimular la atención sostenida a través de la iniciación, mantenimiento y culminación de actividad lúdica en mesa.
- Adaptar el ambiente terapéutico hacia el desarrollo de un juego imaginativo que le permita compartir con pares.

En la Tabla 1 se presentan las características de los medios utilizados en el proceso de intervención con JPJS.

Tabla 1. Características de los medios de intervención

\begin{tabular}{|c|c|}
\hline Encuadre terapéutico & $\begin{array}{l}\text { - Intervención directa con una frecuencia de dos sesiones a la semana, } \\
45 \text { minutos cada una, durante dos meses. Participa en } 20 \text { sesiones de } \\
\text { tipo individual y grupal en el Laboratorio de Juego y Aprendizaje } \\
\text { - Intervención indirecta, en donde se asesora a padres por medio de un } \\
\text { plan de apoyo en casa. }\end{array}$ \\
\hline $\begin{array}{l}\text { Direccionamiento del } \\
\text { terapeuta durante las } \\
\text { actividades }\end{array}$ & $\begin{array}{l}\text { - Establecimiento de contacto visual con el niño brindando instrucciones } \\
\text { cortas, de tipo verbal y demostrativo, antes de iniciar la actividad } \\
\text { planteada para la sesión. } \\
\text { - Utilización de tono de voz alto, simultáneamente se brindan } \\
\text { estímulos táctiles, propioceptivos y vestibulares, los cuales se gradúan } \\
\text { paulatinamente de superficiales a profundos. } \\
\text { - Realimentación verbal en el desarrollo de la actividad y posterior } \\
\text { disminución de la supervisión por parte del terapeuta. } \\
\text { - Reconocimiento de logros constantes al cumplir los pasos de la } \\
\text { actividad propuesta. } \\
\text { Repetición de las instrucciones proporcionadas para la verificación de } \\
\text { la comprensión de las mismas. }\end{array}$ \\
\hline $\begin{array}{l}\text { Ambiente físico } \\
\text { terapéutico }\end{array}$ & $\begin{array}{l}\text { - Modificación del ambiente en donde los equipos de suspensión se } \\
\text { encuentren a una altura aproximada de 1,5 metros del suelo, con el } \\
\text { fin de ir afianzando estímulos vestibulares. } \\
\text { - Regulación de estímulos auditivos y visuales, evitando factores que } \\
\text { promuevan la distracción y facilitando la ejecución de las actividades. } \\
\text { - Adaptación del ambiente hacia el juego imaginativo y de exploración, } \\
\text { teniendo en cuenta los gustos de JPJS; principalmente se ejecutan } \\
\text { las actividades de manera individual y posteriormente se realizan en } \\
\text { compañía de un par. }\end{array}$ \\
\hline $\begin{array}{l}\text { Uso terapéutico del } \\
\text { 'self' }\end{array}$ & $\begin{array}{l}\text { - Relación basada en el respeto a la figura de autoridad. } \\
\text { - } \quad \text { Motivación extrínseca por medio de incentivos verbales y visuales. } \\
\text { - } \text { táctiles y propioceptivos. } \\
\text { - Confianza y seguridad para establecer empatía con el terapeuta. } \\
\text { - } \text { Estabeto de turnos al momento de establecer una conversación. } \\
\text { - Estabiento de contacto visual al brindar realimentación. }\end{array}$ \\
\hline
\end{tabular}




\begin{tabular}{|c|c|}
\hline $\begin{array}{l}\text { Actividades de } \\
\text { intervención directa }\end{array}$ & $\begin{array}{l}\text { - Señalamientos por los cuales se comprendan y acepten las causas y } \\
\text { los efectos de las respuestas brindadas durante la ejecución de las } \\
\text { actividades. } \\
\text { - Estímulos vestibulares con el uso de equipos suspendidos. } \\
\text { - Circuito motor con obstáculos en donde se siguen instrucciones, se } \\
\text { planean y asumen diferentes patrones posturales y de movimiento. } \\
\text { - Circuito con estímulos viso-constructivos, de emparejamiento y } \\
\text { - Asociación de imágenes. } \\
\text { - } \text { colividades motoras finas donde se promueven praxias como y moldeado en mesa. } \\
\text { - Iniciación y culminación de los pasos de la actividad. } \\
\text { - Utilización de la imaginación en piscina de pelotas en compañía de un } \\
\text { par. } \\
\text { Juego cooperativo y de roles con pares a través de circuito sensorial y } \\
\text { motor. }\end{array}$ \\
\hline $\begin{array}{l}\text { Actividades de } \\
\text { intervención indirecta } \\
\text { por medio de plan } \\
\text { casero }\end{array}$ & $\begin{array}{l}\text { La intervención indirecta se ejecuta por medio de un plan casero } \\
\text { entregado a los padres, el cual tiene como propósito brindar } \\
\text { continuidad a la intervención pero en el ambiente familiar del niño. } \\
\text { Algunas de las actividades sugeridas son: } \\
\text { - Juego imitativo (“El Capitán dice") con seguimiento de instrucciones y } \\
\text { mantenimiento de contacto visual. } \\
\text { - Juego imaginativo ("Cuenta la historia") con asociación de imágenes y } \\
\text { - secuenciación. } \\
\text { (“Enogo de planeación y seguimiento de instrucciones } \\
\text { realice planeamiento motor y se ejecuten patrones motores gruesos. }\end{array}$ \\
\hline
\end{tabular}

Fuente. Elaboración propia.

\section{Resultados}

Se realiza un proceso de evaluación inicial y una re-evaluación, a través de observación clínica, utilizando el protocolo antes mencionado, y la aplicación de la batería de evaluación de Escalas de Desarrollo de Merrill Palmer (Roid \& Sampers, 2004). En la Tabla 2 se presentan los resultados comparativos del desempeño antes y después de la intervención descrita en la Tabla 1.

La evaluación realizada después de 20 sesiones de intervención en Terapia Ocupacional evidencia importantes avances en el desempeño ocupacio- nal de JPJS. Según lo reportado por sus padres, el niño desempeña actividades de la vida diaria con supervisión esporádica, participa activamente en su ejecución aunque aún requiere de apoyo para vestirse, alimentarse, en el cepillado de dientes y en el control de esfínteres; actividades en las que la mayoría de niños de su edad, también lo necesitan. Así mismo, se observan cambios en el juego imaginativo, el cual varía de ser solitario a grupal, en donde hay intención de realizar un juego cooperativo interactuando con pares.

En cuanto al seguimiento a realizar con JPJS, se indica a la familia continuar 
Tabla 2. Resultados de la evaluación

\begin{tabular}{|c|c|c|}
\hline Áreas evaluadas & Evaluación inicial & Re-evaluación (20 sesiones después) \\
\hline \multirow{5}{*}{$\begin{array}{l}\text { Destrezas } \\
\text { sensoriales } \\
\text { perceptuales }\end{array}$} & $\begin{array}{l}\text { Identifica pero no responde a } \\
\text { estímulos visuales. }\end{array}$ & $\begin{array}{l}\text { Identifica y responde a estímulos } \\
\text { visuales interpretándolos y asociándolos } \\
\text { a eventos o experiencias previas. }\end{array}$ \\
\hline & $\begin{array}{l}\text { Discrimina y reacciona a } \\
\text { estímulos táctiles. }\end{array}$ & $\begin{array}{l}\text { Discrimina y reacciona a estímulos } \\
\text { táctiles. }\end{array}$ \\
\hline & $\begin{array}{l}\text { Localiza fuente sonora con } \\
\text { frecuentes repeticiones. }\end{array}$ & $\begin{array}{l}\text { Localiza, reconoce y discrimina la } \\
\text { fuente sonora. }\end{array}$ \\
\hline & Reconoce partes de su cuerpo. & Reconoce partes de su cuerpo. \\
\hline & $\begin{array}{l}\text { Se observa inseguridad } \\
\text { gravitacional. }\end{array}$ & $\begin{array}{l}\text { Tolera movimientos en cama elástica y } \\
\text { caballete por más de } 5 \text { minutos. }\end{array}$ \\
\hline \multirow{6}{*}{$\begin{array}{l}\text { Destrezas motoras } \\
\text { y praxis }\end{array}$} & $\begin{array}{l}\text { Disociación de movimientos de } \\
\text { miembros superiores. }\end{array}$ & $\begin{array}{l}\text { Disociación de movimientos de } \\
\text { miembros superiores. }\end{array}$ \\
\hline & $\begin{array}{l}\text { Tono muscular normal en } \\
\text { miembros superiores e inferiores. }\end{array}$ & $\begin{array}{l}\text { Tono muscular normal en miembros } \\
\text { superiores e inferiores. }\end{array}$ \\
\hline & $\begin{array}{l}\text { No mantiene posiciones por más } \\
\text { de } 2 \text { minutos. }\end{array}$ & $\begin{array}{l}\text { Asume y mantiene patrones posturales } \\
\text { por más de } 5 \text { minutos. }\end{array}$ \\
\hline & $\begin{array}{l}\text { Realiza actividades de } \\
\text { coordinación dinámica general } \\
\text { tales como: patear, lanzar con } \\
\text { una y dos manos, atajar con dos } \\
\text { manos y pies; y de coordinación } \\
\text { dinámica manual tales como: } \\
\text { aplaudir, cortar plastilina, colorear, } \\
\text { recortar, rasgar, encajar, picar } \\
\text { con agarre y arrugar, ideando } \\
\text { y ejecutando cada una de las } \\
\text { acciones. }\end{array}$ & $\begin{array}{l}\text { Realiza actividades de coordinación } \\
\text { dinámica general tales como: patear, } \\
\text { lanzar con una y dos manos, atajar con } \\
\text { dos manos y pies; y de coordinación } \\
\text { dinámica manual tales como: aplaudir, } \\
\text { cortar plastilina, colorear (en donde se } \\
\text { observa una variación entre agarre y } \\
\text { pinza), recortar, rasgar, encajar, picar } \\
\text { con agarre y arrugar. Dichas actividades } \\
\text { son realizadas por ideación, planeación } \\
\text { y ejecución. }\end{array}$ \\
\hline & $\begin{array}{l}\text { En motricidad gruesa y fina } \\
\text { presenta una edad equivalente } \\
\text { a } 31 \text { meses según la escala de } \\
\text { Merrill Palmer. }\end{array}$ & $\begin{array}{l}\text { En motricidad gruesa y fina presenta } \\
\text { una edad equivalente a } 36 \text { meses según } \\
\text { la escala de Merrill Palmer. }\end{array}$ \\
\hline & $\begin{array}{l}\text { En coordinación visomotora } \\
\text { presenta una edad de } 28 \text { meses. }\end{array}$ & $\begin{array}{l}\text { En coordinación visomotora presenta } \\
\text { una edad de } 37 \text { meses. }\end{array}$ \\
\hline \multirow{3}{*}{$\begin{array}{l}\text { Destrezas de } \\
\text { regulación } \\
\text { emocional }\end{array}$} & $\begin{array}{l}\text { Baja tolerancia a la frustración } \\
\text { frente a situaciones como la } \\
\text { separación de los padres y la } \\
\text { alteración de rutinas diarias. }\end{array}$ & $\begin{array}{l}\text { Sigue rutinas establecidas en el } \\
\text { ambiente terapéutico. } \\
\text { Tolera separación de padres. }\end{array}$ \\
\hline & $\begin{array}{l}\text { Irritabilidad, agresividad y poca } \\
\text { autorregulación. }\end{array}$ & $\begin{array}{l}\text { Manifiesta y comunica sus emociones } \\
\text { sin llanto. }\end{array}$ \\
\hline & $\begin{array}{l}\text { Presenta una edad de } 26 \text { meses } \\
\text { según la escala de Merrill Palmer. }\end{array}$ & $\begin{array}{l}\text { Presenta una edad de } 35 \text { meses según la } \\
\text { escala de Merrill Palmer. }\end{array}$ \\
\hline
\end{tabular}




\begin{tabular}{|c|c|c|}
\hline \multirow{5}{*}{$\begin{array}{l}\text { Destrezas } \\
\text { cognitivas }\end{array}$} & $\begin{array}{l}\text { Dificultad en procesos } \\
\text { atencionales. } \\
\text { Soluciona problemas sencillos. }\end{array}$ & $\begin{array}{l}\text { Mantiene atención en actividades } \\
\text { de mesa por más de } 10 \text { minutos en } \\
\text { posición sedente. }\end{array}$ \\
\hline & $\begin{array}{l}\text { Comprende y sigue instrucciones } \\
\text { con repeticiones constantes de } \\
\text { estas. }\end{array}$ & Sigue cuatro instrucciones consecutivas. \\
\hline & $\begin{array}{l}\text { Presenta una edad equivalente } \\
\text { a } 29 \text { meses según la escala de } \\
\text { Merrill Palmer. }\end{array}$ & $\begin{array}{l}\text { Presenta una edad equivalente a } 37 \\
\text { meses según la escala de Merrill Palmer. }\end{array}$ \\
\hline & $\begin{array}{l}\text { En memoria presenta una edad de } \\
28 \text { meses. }\end{array}$ & $\begin{array}{l}\text { En memoria presenta una edad } \\
\text { equivalente a } 36 \text { meses. }\end{array}$ \\
\hline & $\begin{array}{l}\text { En velocidad de procesamiento } \\
\text { presenta una edad de } 31 \text { meses. }\end{array}$ & $\begin{array}{l}\text { En velocidad de procesamiento presenta } \\
\text { una edad de } 41 \text { meses. }\end{array}$ \\
\hline \multirow{6}{*}{$\begin{array}{l}\text { Destrezas de } \\
\text { comunicación y } \\
\text { sociales }\end{array}$} & $\begin{array}{l}\text { Realiza pero no mantiene contacto } \\
\text { visual. }\end{array}$ & $\begin{array}{l}\text { Realiza y mantiene contacto visual } \\
\text { durante una conversación. }\end{array}$ \\
\hline & $\begin{array}{l}\text { Comunica sus intereses de forma } \\
\text { no verbal. }\end{array}$ & $\begin{array}{l}\text { Comunica sus intereses y emociones } \\
\text { acertadamente y sin llanto. }\end{array}$ \\
\hline & $\begin{array}{l}\text { Inicia pero no mantiene una } \\
\text { conversación. }\end{array}$ & $\begin{array}{l}\text { Inicia y mantiene conversaciones } \\
\text { prolongadas. }\end{array}$ \\
\hline & No se relaciona con pares. & Entabla relación con pares. \\
\hline & $\begin{array}{l}\text { Pocas veces realiza frases de cinco } \\
\text { o más palabras. }\end{array}$ & $\begin{array}{l}\text { Establece conversaciones concretas con } \\
\text { frases de cinco palabras. }\end{array}$ \\
\hline & $\begin{array}{l}\text { Presenta una edad equivalente } \\
\text { a } 25 \text { meses según la escala de } \\
\text { Merrill Palmer. }\end{array}$ & $\begin{array}{l}\text { Presenta una edad equivalente a } 31 \\
\text { meses según la escala de Merrill Palmer. }\end{array}$ \\
\hline \multirow[t]{2}{*}{ Autocuidado } & $\begin{array}{l}\text { Realiza actividades como: vestido } \\
\text { independientemente, cepillado } \\
\text { con supervisión, baño con } \\
\text { supervisión, control de esfínteres y } \\
\text { alimentación. }\end{array}$ & $\begin{array}{l}\text { Realiza actividades como: vestido } \\
\text { independientemente, cepillado con } \\
\text { supervisión, baño con supervisión, } \\
\text { control de esfínteres y alimentación. }\end{array}$ \\
\hline & $\begin{array}{l}\text { Presenta una edad de } 39 \text { meses } \\
\text { según la escala de Merrill Palmer. }\end{array}$ & $\begin{array}{l}\text { Presenta una edad de } 39 \text { meses según la } \\
\text { escala de Merrill Palmer. }\end{array}$ \\
\hline
\end{tabular}

Fuente. Elaboración propia.

con proceso terapéutico en el servicio, con la misma frecuencia de intervención y realizando semestralmente evaluaciones del desempeño del niño.

\section{Discusión}

El caso de JPJS implica que el equipo de terapeutas ocupacionales realice una revisión minuciosa de diferentes aspectos teóricos y prácticos, que les permitan cumplir con los retos de la intervención y los objetivos que se pretenden alcanzar con el niño, a nivel de su desempeño ocupacional.

En primera instancia, se hace una aproximación teórica al diagnóstico con el cual el niño llega al servicio de Terapia Ocupacional; para ello se revisan planteamientos como los de Kadar, McDonald \& Lentin (2012) y los de Ortiz (2014), autores que afirman 
que las alteraciones sensoriales en las personas con diagnóstico de TEA son frecuentes, y que estas a su vez afectan el desempeño en las actividades de la vida diaria, así como sus habilidades y respuestas al entorno, lo que genera aislamiento social y mayores dificultades en el desempeño ocupacional. Posterior a esto, se determina la importancia que dan estos autores a que las intervenciones frente a dicho diagnóstico se centren en la integración sensorial.

Con base en lo anterior, se revisan los planteamientos de Ayres respecto al proceso de integración sensorial, quien lo define como un proceso neurológico que organiza e interpreta las sensaciones recibidas del ambiente, para permitir usar el cuerpo efectivamente en el entorno. Aquello es necesario para que la persona responda adaptativamente y se faciliten procesos de aprendizaje e interacción con el ambiente, desempeñándose adecuadamente en su entorno (Beundry, 2013). Lo anterior brinda información fundamental para establecer objetivos de intervención claros.

Así, con el fin de encaminar de manera efectiva el plan de tratamiento con JPJS, se determinó basar la intervención en la integración sensorial. Autores como Watling \& Dietz (2007), plantean que la intervención basada en integración sensorial permite que la persona con TEA realice diferentes tipos de movimiento por medio del juego y, a partir de estos, reciba estímulos sensoriales que debe procesar, modular e integrar de manera adecuada para responder adaptativamente frente a la actividad lúdica; además afirman que, la intervención centrada en este tipo de actividades, mejora el contacto visual, la comunicación y el comportamiento social, todo esto proporciona soporte teórico relevante para dar solución a las necesidades evidenciadas en el caso.

Por otra parte, autores como CaseSmith, Weaver \& Fristad (2014) afirman que las intervenciones dirigidas a la integración sensorial y realizadas por medio de actividades de juego e interacciones, permiten que el niño brinde respuestas adaptativas como lo son los comportamientos organizados, el desarrollo de habilidades sociales, la planeación motora y el desarrollo de habilidades perceptuales. Así mismo, Parham et al. (2011) mencionan que dichas actividades, dirigidas a la integración sensorial, implican una estimulación táctil, vestibular o propioceptiva, que permite mayor autorregulación y conciencia sensorial y de movimiento para el desarrollo de nuevos comportamientos motores y la organización de movimientos en tiempo y espacio.

Con base en los referentes antes enunciados, se plantean para JPJS actividades como la realización de circuitos motores en donde se brindan estímulos vestibulares suaves en plano horizontal, siendo posible que alcance niveles de alerta adecuados, contacto visual por periodos prolongados de tiempo y el seguimiento de instrucciones cortas. De otra parte, el niño recibe estímulos táctiles en diferentes segmentos corporales a través de texturas lisas y maleables, y estímulos propioceptivos, como el contacto físico firme en las articulaciones proximales, para 
favorecer la conciencia corporal y la autorregulación. Todo esto permite alcanzar logros significativos.

Adicionalmente, se ve la necesidad de fomentar los procesos de adaptación del niño; a este respecto, autores como Durante \& Noya (1998) mencionan que el modelo de habilidades adaptativas propuesto por Ann Mosey en 1968, ofrece elementos conceptuales que favorecen la intervención en estos casos, ya que al proveer experiencias de participación en diversos ambientes, el desempeño ocupacional puede mejorar. Sin embargo, es necesario considerar el desarrollo de habilidades de aprendizaje y habilidades evolutivas, junto con el trabajo de componentes como la integración sensorial, la función cognitiva, la función psicosocial y la interacción social, puesto que estos favorecen la planeación, la ejecución de tareas y la satisfacción de necesidades.

Teniendo en cuenta lo anterior, se decide involucrar de manera constante en las diferentes actividades, acciones que cumplan con los fines planteados desde dicho modelo. Ello permite observar en el niño mayores destrezas en la ejecución de acciones que impliquen un proceso de adaptación.

Así, de acuerdo a lo manifestado por los padres, JPJS aumenta su tolerancia a la frustración y muestra una mayor adaptación para identificar dificultades al momento de responder a las exigencias del entorno y a los retos que se le presentan cotidianamente. Se observan además avances significativos en su capacidad perceptiva motriz, caracterizada por recibir y seleccionar la información vestibular, propioceptiva y táctil, logrando una respuesta funcional a partir de esta (Donohue, 1990); también en la capacidad cognitiva, dirigida a percibir, representar, y organizar la información sensorial para resolver problemas; en la capacidad de interacción grupal, que se ocupa de participar e implicarse en relaciones diádicas y de grupos; y en la capacidad de auto identidad, que responde a la habilidad de percibirse como un ser autónomo y con permanencia y continuidad en el tiempo.

Para el alcance y desarrollo de todos estos aspectos, el terapeuta ocupacional realiza intervenciones individuales y grupales a través del juego. Inicialmente se emplea el juego paralelo, basado en sus intereses, lo que le permite a JPJS adquirir confianza y establecer empatía con el terapeuta y con sus pares. Posteriormente el niño inicia la ejecución de juego proyectivo, mostrándose interesado en compartir y cooperar dentro del contexto lúdico, además de promover el disfrute con sus compañeros de juego. Finalmente el niño realiza un juego cooperativo, en donde conoce y respeta las normas y límites de la actividad, cumple con un rol específico y participa para satisfacer sus necesidades de disfrute y placer frente al juego, así como las de los demás. Se evidencia entonces que el niño desarrolla habilidades y capacidades que le permiten generar respuestas adaptativas en diferentes contextos.

El entorno fue otro factor fundamental en el proceso de intervención de JPJS. De acuerdo con Gilfoyle et al. (1990), el entorno es todo aquello con lo que el individuo interactúa y toma 
lugar dentro las dimensiones de espacio y tiempo. La dimensión espacial es todo lo que rodea al individuo, el espacio ocupado por su propio cuerpo, el de las demás personas y los objetos dentro de un área. El tiempo o los aspectos temporales son definidos por estos autores como la duración y regulación de las secuencias motoras realizadas por la persona dentro del espacio. El desarrollo del movimiento y la forma de moverse, tienen un gran vínculo con la naturaleza del entorno y la interacción del individuo con este. Teniendo en cuenta lo anterior, se establecen actividades donde el niño ponga en función su cuerpo frente las exigencias del entorno, permitiéndole a su vez poner en juego destrezas a nivel motor, cognitivo - perceptual y de interacción - comunicación.

Para el manejo del entorno se busca dar un contexto espaciotemporal que cumpla con sus cuatro funciones: mantenimiento, que sirve para dar contención al niño y brindarle soporte al cuerpo; facilitador, que provee el curso de estimulación, excitación, intención y motivación del movimiento; desafío, que ayuda al niño a alcanzar mayores niveles de adaptación; e interacción, con la que se busca promover las adecuadas relaciones y respuestas del niño con el entorno. A través de estas funciones los individuos adquieren una conciencia espaciotemporal, responden a las exigencias del ambiente, conocen las reglas y límites establecidos, se fomentan la interacción social y el desarrollo de la flexibilidad del comportamiento o la adaptabilidad para una interacción exitosa con el entorno (Gilfoyle et al.,1990).
Un tercer aspecto considerado es la adaptación, definida como un proceso dinámico que permite ampliar el repertorio de movimientos y actividades del niño. A través de la adaptación, el desarrollo de los movimientos es más complejo, de tal manera que provee al individuo habilidades para aumentar la interacción con el entorno. La adaptación es definida como los ajustes en los procesos corporales, que se llevan a cabo dentro de la correlación de espacio y tiempo (Gilfoyle et al.,1990).

De acuerdo con Gilfoyle et al. (1990), para llegar a una adaptación es importante el proceso sensorio-motor, el cual incluye cuatro componentes: asimilación, acomodación, asociación y diferenciación. La asimilación es el proceso de recibir la información; la acomodación, la capacidad que tiene el sujeto de modificar posturas y ajustar posiciones para dar respuesta a la información recibida; la asociación es un proceso organizado que favorece la relación entre la información sensorial y la motora, con las experiencias previamente adquiridas; y la diferenciación es el proceso de discriminar aquellos elementos esenciales y comportamientos específicos, que son pertinentes para responder a una situación, de aquellos que no lo son, para poder así modificarlos o alternar el comportamiento.

En JPJS estos cuatro componentes se manifiestan paulatinamente, a medida que va alcanzando las habilidades requeridas para el proceso de adaptación espacio temporal. La asimilación se caracteriza por recibir información sensorial del ambiente y producir respuestas motoras que permiten dar alcance a la acomodación, evidente 
en el control postural que adquiere cuando se relaciona con objetos o estímulos proporcionados en diferentes planos. La asociación se observa cuando el niño responde asertivamente a los juegos que previamente se le han planteado, permitiendo que discrimine cuáles elementos del juego y de sus habilidades son pertinentes para alcanzar los requerimientos propuestos por el terapeuta, manifestándose a su vez el componente de diferenciación.

El último aspecto considerado corresponde al continuo espiral, que se explica a partir de la adaptación de patrones primitivos de postura y movimiento, a mayor nivel y complejidad en el desarrollo de destrezas. El proceso de continuo espiral incluye: entrada sensorial, respuesta motora y realimentación sensorial; la asimilación está relacionada con la entrada de la información sensorial, la acomodación vista como una respuesta motora y la asociación/diferenciación de información para facilitar futuros niveles de desempeño. Cuando el entorno provee una nueva experiencia, el niño se adapta gracias a los patrones de comportamiento previamente adquiridos e integra la información sensorial, a partir de los estímulos proporcionados en esta nueva situación. A través de la integración, el sistema sensorial organiza la información desde la experiencia (Gilfoyle et al., 1990).

\section{Conclusión}

La intervención de Terapia Ocupacional con JPJS demuestra que el proceso terapéutico basado en objetivos relacionados con la modulación sensorial, la autorregulación, el seguimiento de instrucciones, los procesos atencionales y la interacción con pares, es fundamental para lograr avances en el desempeño cotidiano de niños y niñas con estas características.

Por otro lado, se brinda a otros terapeutas ocupacionales, a partir de una experiencia profesional efectiva y contextualizada en el sistema de salud colombiano, información útil para orientar sus planes de tratamiento. Esto llama además la atención sobre la necesidad de documentar experiencias de este tipo de manera rigurosa, para así dar soporte científico a las intervenciones realizadas en el país.

La vinculación y la participación de la familia y los cuidadores en el proceso de intervención es fundamental, ya que son actores centrales en la vida cotidiana de los niños y las niñas; esto implica un compromiso importante del terapeuta para alcanzarlas. Este caso se caracterizó por el compromiso de la familia en las intervenciones propuestas por el terapeuta y en la transferencia de las orientaciones en casa por medio de plan de apoyo, lo que permitió logros en la rutina familiar de JPJS.

A partir de los avances en el proceso de intervención, se recomienda a la familia solicitar una revaloración por parte de Neuropediatria, para hacer seguimiento al proceso de neurodesarrollo y determinar la pertinencia de intervenciones adicionales por parte de otras disciplinas.

La evidencia consultada y este caso en particular muestran que, el uso de referentes teóricos tales como el modelo de habilidades adaptativas, el enfoque de adaptación espacio temporal y el 
enfoque de integración sensorial, son fundamentales para la planeación e intervención dirigidas a impactar el desempeño ocupacional de niños y niñas.

\section{Referencias}

Arntsein. S. (1969). A Ladder of Citizen Participation. Journal of the American Planning Association; 35 (4). 216-224.

Asociación Americana de Psiquiatría. (2014). DSM-5 Manual Diagnóstico y Estadístico de Trastornos Mentales. Barcelona (España): Elsevier Masson.

Beundry. I. (2013). El enfoque de la integración sensorial de la doctora Ayres. TOG; 10(17).1-11.

Case-Smith, J., Weaver, L. L., \& Fristad, M. A. (2014). A systematic review of sensory processing interventions for children with autism spectrum disorders. Autism, 1362361313517762.

Donohue. M. (1999). Theoretical bases of Mosey's group interaction skills. Occupational Therapy International; 6 (1). 35-51

Durante. P., y Noya. B.(1998). Terapia Ocupacional en salud mental: principios y practica. España: Masson.

Gilfoyle. E., Grady. A., \& Moore. J.(1990). Children adapt: a theory of sensorimotorsensory development. Thorofare (Nueva Jersey, Estados Unidos): Slack.

Howell, D. M., Wittman, P., \& Bundy, M. B. (2012). Interprofessional clinical education for occupational therapy and psychology students: A social skills training program for children with autism spectrum disorders. Journal of Interprofessional Care, 26(1), 49-55. doi: 10.3109/13561820.2011.620186

Kadar, M., McDonald, R., Lentin, P. (2012). Evidence-Based in Occupational Therapy Services for children with autism spectrum disorder in Victoria, Australia. Australian Occupational Therapy Journal. 59(10). 284293

Matsushima, K., \& Kato, T. (2013). Social Interaction and Atypical Sensory Processing in Children with Autism Spectrum Disorders. Hong Kong Journal of Occupational Therapy, 23(2), 89-96. doi: 10.1016/j. hkjot.2013.11.003

Mulas, F., Ros-Cervera, G., Millá, M. G., Etchepareborda, M. C., Abad, L., y Téllez de Meneses, M. (2010). Modelos de intervención en niños con autismo. Rev Neurol, 50(S03), 077-S084.

Ortiz, H. (2014).Terapia de integración sensorial en niños con Trastorno de Espectro Autista.TOG. 11(19). 1-13

Parham, L. D., Roley, S. S., May-Benson, T. A., Koomar, J., Brett-Green, B., Burke, J. P., et al. (2011). Development of a fidelity measure for research on the effectiveness of the Ayres sensory integration intervention. The American Journal of Occupational Therapy, 65, 133-142.

Peñaranda. A. (2007). Instrumento de evaluación de destrezas de ejecucion del Laboratorio de juego y aprendizaje. Universidad del Rosario. Colombia

Rivero, P. F. P., y Garrido, L. M. M. (2014). Perfiles cognitivos en el Trastorno Autista de Alto Funcionamiento y el Síndrome de Asperger. Revista CES Psicología, 7(1), 141 155. doi: 10.1007/s10803-011-1219-8

Roid. G, \& Sampers, J. (2004). Escalas de desarrollo Merrill-Palmer. Illiniois: T. Ediciones.

Talero, C., Rodriguez, M., De La Rosa, D., Morales, G., \& Velez-Van-Meerbeke, A. (2012). Profile of children and adolescents with autism spectrum disorders in an institution in Bogota, Colombia. Neurología, 27(2), 90-96. doi: 10.1016/j. nrl.2011.03.005

Ventura-Juncá, P., \& Tapia, J. L. (2004). El recién nacido normal: Atención inmediata, cuidado de transición y puerperio. Tapia $J L$, Ventura Junca P. Manual de Neonatologia. Santiago de Chile. Mediterráneo 1992: 503, 910.

Watling, R., \& Dietz, J.(2007). Immediate Effect of Ayres's Sensory Integration-Based Occupational Therapy Intervention on Children With Autism Spectrum Disorders. American Journal of Occupational Therapy. 61(5). 574-583. 